\title{
The Application of Coherent Local Time for Optical Time Transfer and the Quantification of Systematic Errors in Satellite Laser Ranging
}

\author{
K. Ulrich Schreiber ${ }^{1}$ (D) Jan $\operatorname{Kodet}^{1}$ (D)
}

Received: 19 November 2016 / Accepted: 7 December 2017 / Published online: 20 December 2017

(C) The Author(s) 2017. This article is published with open access at Springerlink.com

\begin{abstract}
Highly precise time and stable reference frequencies are fundamental requirements for space geodesy. Satellite laser ranging (SLR) is one of these techniques, which differs from all other applications like Very Long Baseline Interferometry (VLBI), Global Navigation Satellite Systems (GNSS) and finally Doppler Orbitography and Radiopositioning Integrated by Satellite (DORIS) by the fact that it is an optical two-way measurement technique. That means that there is no need for a clock synchronization process between both ends of the distance covered by the measurement technique. Under the assumption of isotropy for the speed of light, SLR establishes the only practical realization of the Einstein Synchronization process so far. Therefore it is a powerful time transfer technique. However, in order to transfer time between two remote clocks, it is also necessary to tightly control all possible signal delays in the ranging process. This paper discusses the role of time and frequency in SLR as well as the error sources before it address the transfer of time between ground and space. The need of an improved signal delay control led to a major redesign of the local time and frequency distribution at the Geodetic Observatory Wettzell. Closure measurements can now be used to identify and remove systematic errors in SLR measurements.
\end{abstract}

Keywords Satellite laser ranging · Optical time transfer - Accurate time and frequency

High Performance Clocks with Special Emphasis on Geodesy and Geophysics and Applications to Other Bodies of the Solar System

Edited by Rafael Rodrigo, Véronique Dehant, Leonid Gurvits, Michael Kramer, Ryan Park, Peter Wolf and John Zarnecki

K.U. Schreiber

ulrich.schreiber@bv.tum.de

1 FESG, Geodätisches Observatorium Wettzell, Technical University of Munich,

Sackenrieder Str. 25, 93444 Bad Kötzting, Germany 
Fig. 1 Simplified illustration of the satellite laser ranging technique. All signal delays, which are not part of either the measured distance or the calibration range, cancel out entirely

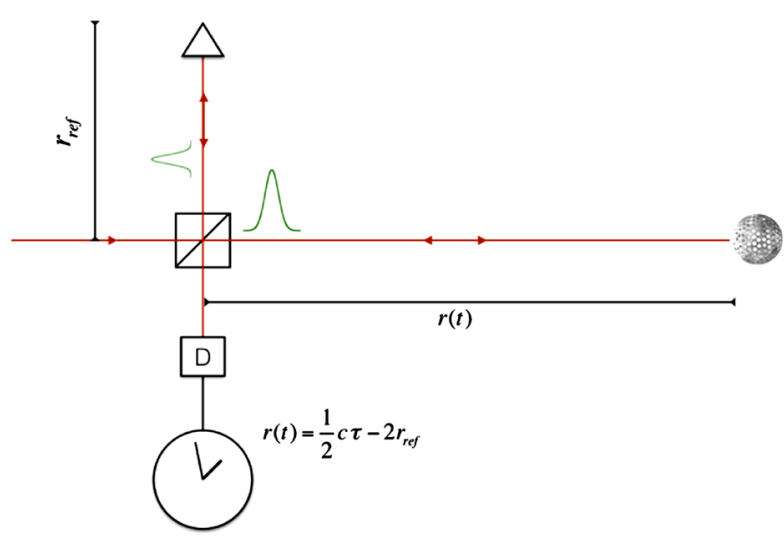

\section{The Technique of Satellite Laser Ranging}

\subsection{A Two-Way Concept of Measuring the Time of Flight}

Satellite Laser Ranging (SLR) establishes the distance between a fixed point (station) on the ground and a satellite target, utilizing a time of flight measurement of short laser pulses. It is unique in the ensemble of space geodetic measurement techniques, because it operates in the optical regime and it realizes a two-way measurement concept. This means that there is no need to synchronize the transmitter and receiver clocks in a similar fashion as necessary for the VLBI and the GNSS techniques, because the time of flight of short mode-locked laser pulses is established by the same local clock, so that a clock offset cancels out. Although the individual instrumental components of SLR require challenging technologies, the underlying measurement concept is very simple and also has a clear unambiguous geometrical meaning (Degnan 1993). Figure 1, drawn in the form reminiscent of a Michelson interferometer, illustrates this process. Short laser pulses are generated by a mode-locked laser and a small portion of this signal is directed to the start detector, timing the epoch $t_{1}$ of the outgoing signal. Technically this requires a beam splitter and in the interest of simplicity, we have placed this device at the reference point of our measurement system. Most of the laser pulse intensity is steered towards the satellite of interest by the help of an optical telescope not shown in the functional diagram. The laser pulses are back reflected from a suitable retro reflector array and also timed as $t_{2}$ on the local clock of the SLR station. With $\tau=t_{2}-t_{1}$ the distance to the satellite is obtained as $r(t)=\frac{1}{2} c \tau-2 r_{\text {ref }}$, where $r_{\text {ref }}$ is the contribution of the reference arm, providing a range of precisely known length. We note that for each laser shot this detection process happens at an delayed instance, which is not a priori known. It is caused by the travel time of the laser pulses and the electrical signals inside the system. Hence it is necessary to compensate these extra delays by performing a reference measurement over an exactly known distance. In our simplified diagram this corresponds to the reference arm of the sketched interferometer. From the idealized situation shown in Fig. 1 one can see that every delay cancels out. In the real instrumentation a few compromises are necessary for technical reasons, so that the ideal situation is only approximated.

SLR systems typically operate solid state lasers, where the active material is Nd:YAG (an yttrium aluminium garnet crystal doped with neodymium), which produce short laser pulses with a pulse width of the order of $10 \mathrm{ps} \leq \tau \leq 200 \mathrm{ps}$ at a wavelength of $1.064 \mu \mathrm{m}$. Optical frequency doubling places the wavelength at $532 \mathrm{~nm}$, which is in the middle of the visible 
part of the optical spectrum, where photodetectors are most sensitive with quantum efficiencies exceeding $70 \%$. When the laser pulse is generated it is time tagged as $t_{0}$ on the local clock and that determines the epoch of the measurement. The laser pulse is then guided to a telescope, which can be steered such that it points to a geodetic satellite and tracks it along its orbit, while the satellite is above the horizon of the observing station. The intensity of the satellite reflection is highly variable and more than 10 orders of magnitude smaller when it hits the detector. Therefore a high detector gain is required to produce a measurable signal response at $t_{1}$. For practical reasons all currently operating SLR systems separate the process of the range measurement from the process of the reference measurement. Sometimes the calibration of the SLR system is only repeated every hour or even less often. The time interval $\Delta t$ of the measurement between the epoch of the return signal and the epoch of the laser fire event contains a number of system and concept dependent delays, which have to be removed before the exact distance between the reference point on the ground (namely the intersection point of the horizontal and the vertical axis of the telescope) and the reflector array on the satellite can be derived.

$$
\left.\Delta t_{e}^{s}\right|_{t_{0}}=\frac{2}{c}\left(\rho_{e}^{s}+\delta \rho_{a t m}+\delta \rho_{r e l}\right)+\frac{1}{c} \delta \rho_{s y s}+\epsilon_{e}^{s}
$$

Here $\left.\Delta t_{e}^{s}\right|_{t_{0}}$ is the time interval between laser fire and detection at the epoch $t_{0}, \rho_{e}^{s}$ is the distance between the reference points, $\delta \rho_{\text {atm }}$ the atmospheric contribution to the path delay, $\delta \rho_{\text {rel }}$ accounts for relativistic effects and $\delta \rho_{s y s}$ is the system delay. The value $c$ is the vacuum velocity of light and $\epsilon_{e}^{s}$ corresponds to stochastic measurement errors. In order to relate the optical distance measurement to the center of mass of the satellite, the eccentricity vector between the center of mass of the satellite and the reflector array has to be applied. While this correction is fairly simple for spherically shaped satellites with laser reflectors all over the surface like for the satellites LAGEOS, STARLETTE or ETALON, this correction can be very tedious and attitude dependent for irregular shaped Earth observing satellites with an reflector array somewhere at a corner. This problem can be even worse when the satellite is losing mass throughout its mission for example by burning fuel.

\subsection{The Importance of Time and Frequency in SLR}

For SLR measurements we have to distinguish between the time used in the context of establishing the epoch of an individual range measurement and the actual round trip time interval measurement of the laser pulse. The former is related to UTC time, while the latter is obtained from the frequency of the oscillator of the clock. This measurement epoch is associated with the position of the satellite along the orbit and the requirements for the accuracy of this value are not particularly high. A low Earth orbiting satellite with velocities of the order of $10 \mathrm{~km} / \mathrm{s}$, changes its position approximately by $1 \mathrm{~cm}$ in $1 \mu \mathrm{s}$. So, if the station clock is offset from true UTC by several $\mu$ s, the satellite appears shifted along its orbit. Radiation pressure and more importantly a deceleration from the gas pressure of the residual atmosphere are also affecting the satellite motion and have to be modelled in the analysis process. Therefore the correct measurement epoch (station clock offset) is finally established from the non-linear least squares adjustment procedure from the combined measurements of a network of SLR stations.

The accurate measurement of the round trip time of the light is far more critical for the measurement process than the epoch, because the error margin of the established distance depends on the resolution of the timing process of measuring the time of flight of a laser 
Fig. 2 The variation of the time delay between the rising edges of the pulse per second signals, derived from the same maser in 2 different buildings. The inset illustrates schematically the detection timing jitter in the presence of unavoidable phase noise. The arrow indicates the detection threshold and the greyed out area illustrates the uncertainty in timing

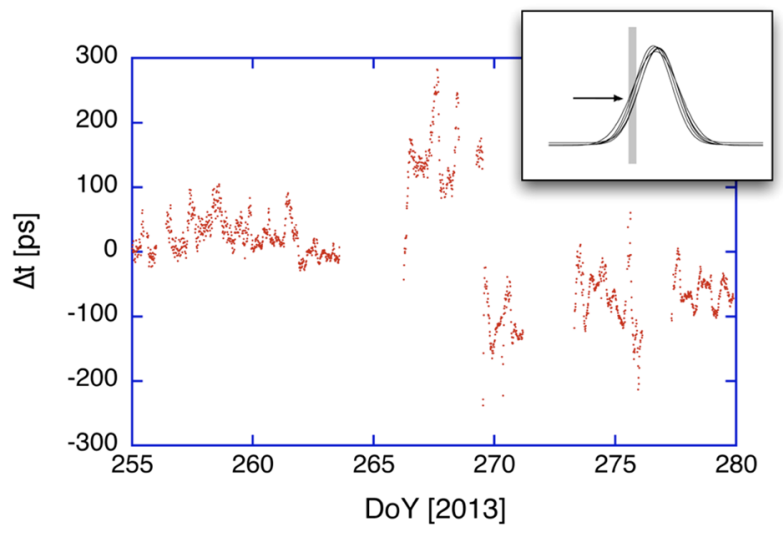

pulses. This in turn is depending on the pulse width of the laser, the bandwidth of the detector and signal conditioning unit and the granularity of the timescale of the clock used in the timing process. Modern detectors provide signal rise times of about $100 \mathrm{ps,} \mathrm{a} \mathrm{typical} \mathrm{laser}$ generates pulses in the range of $30 \mathrm{ps}$ FWHM and the smallest division of modern event timing devices is just below of 3 ps. As the measurement precision improves with averaging, it is therefore desirable to operate SLR systems with a high repetition rate of $1-2 \mathrm{kHz}$ in order to exploit averaging from a high number of range measurements. However, the potential gain from going to higher repetition rates is not indefinite, because the range measurements do not follow a Gaussian distribution as outlined in the next section. As a consequence of such highly developed subsystems we find a measurement precision of the order of $3 \mathrm{~mm}$ for a modern SLR systems.

\section{Error Sources and Limitations in SLR}

\subsection{Temperature Related Effects on the Timing Electronics}

While a single shot precision of less than $15 \mathrm{ps}$ is routinely achieved by several SLR systems, the respective obtained accuracy for the reconstruction of the satellite orbit is not better than 20-45 ps. This is not only because of some fundamental short-comings of the current realization of the SLR technique (Appleby et al. 2016). Apart from small deficiencies in the modelling of the satellite dynamics and the contribution of the chosen frame of reference a number of small technically induced systematic biases need to be addressed, so that this complex error budget eventually can be disentangled. Together with stability and bandwidth related issues of comparable size within the VLBI technique, this leads to an apparent discrepancy for the scale of the ITRF of the order of 2 parts in $10^{9}$. Several mechanisms are contributing to this error budget. Time corresponds to the integral of a reference frequency of a stable clock. Timing signals therefore are typically derived from the leading edge of electrical pulses. In order to obtain a high measurement resolution this ideally requires an infinite steep slope, which is practically not achievable. The maximum bandwidth obtainable from the available technology of electronic circuitry is between 1 and $10 \mathrm{GHz}$. Therefore the actual timing accuracy for the threshold detection is highly dependent on the stability of the ground potential and the bandwidth of the measurement channel, as well as the phase noise on the rising edge of the signal. Figure 2 illustrates this for a comparison of the pulse 
Fig. 3 The variation of the measured time interval along a short fixed local calibration range for a constant optical signal level. The variability of the range is due to the unavoidable and variable signal propagation delay inside the electronic units of the ranging system

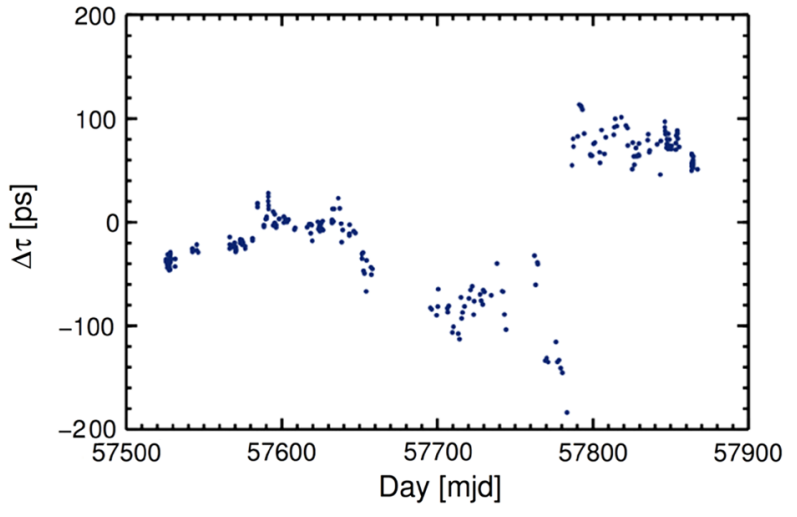

per second (PPS) square wave signals between two buildings approximately $60 \mathrm{~m}$ apart. The inset in Fig. 2 illustrates how fluctuations in ground potential and phase noise map into time in the presence of a bandwidth limited detection. However, the most dramatic impact on the displayed measurement time series is caused by the temperature dependence of the transition delay in the applied electronic circuitry. Evidence to this is the diurnal signature of measured time delays in the first part of Fig. 2. Technical problems of the air conditioning system and human activity around the clock area led to the much larger excursions for the delay of the clock comparison, shown in the second part of the plot, before the delay returns to the original pattern towards the end of this 25 day observation period.

While Fig. 2 illustrates the difficulty to obtain a long term stable delay comparison between two buildings, Fig. 3 depicts the achievable long term system stability of the calibration of a single SLR station. Each data point in the diagram corresponds to the mean value of a measurement series, where the laser is fired a few thousand times at a flat calibration target. For each shot the epoch of the laser fire event is taken by the start detector. Shortly afterwards the calibration stop is obtained from the telescope detector, when the laser pulse is returned to the system after passing through the reference range. Since the reference range and the detectors are held constant, Fig. 3 provides the stability of the timing circuitry. Over a period of one year, one can see a peak to peak variation of about $300 \mathrm{ps}$ in the electronic circuitry of the system delay. The main contributor for this variability are temperature induced delays in the electronics. For most of the time the change in value from one day to the next is small, however there are also some sudden jumps in the data. Since the start and stop signals are timed on the same clock, a clock offset does not affect the measurements, because it is common to both timing processes and disappears in the difference. Keeping in mind that the entire measurement error should not exceed a few ps, this also means that not only the clock must be the same, but also the electronic signal path for both, laser fire (start signal) and echo detection (stop signal). A different signal path for timing the start and the stop event inside the timer hardware of an SLR system introduces an unknown and due to the discussed temperature sensitivity slowly variable delay, which consequently compromises the accuracy of the measurement.

\subsection{Delays from the Conversion from the Optical to the Electrical Domain}

So far we have discussed variable system delays, which are mainly caused by temperature related variations in the transfer of broadband timing signals along the electronic circuitry of the event timing system. While this type of system delay is caused on the signal path 
Fig. 4 This figure illustrates the dependence of the conversion delay of an optical input signal of a photodiode to an electrical output signal on the input signal intensity. Over 5 orders of magnitude of variation of the input intensity caused a non-linear change in detector timing delay of more than 500 ps

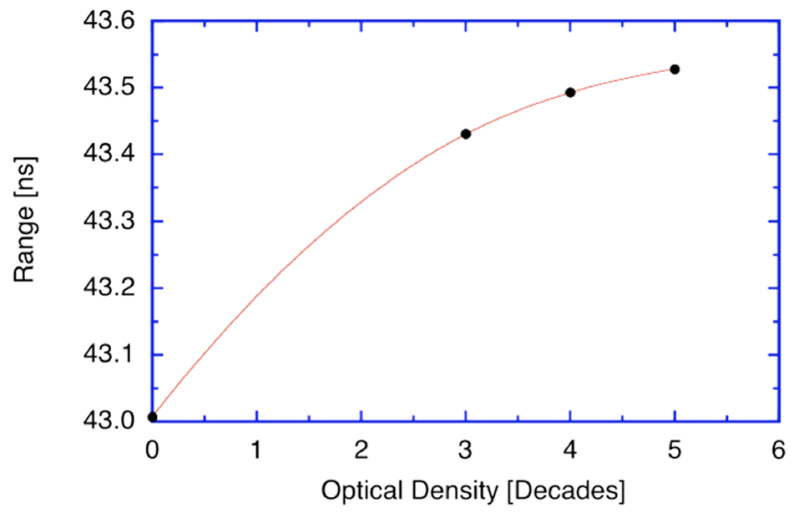

between the various components of the ranging system, the optical echo detection process also contributes to the error budget. The response of a bound electron to the electromagnetic force of light is fast but not instantaneous (Hassan et al. 2016). While the resultant delay is on the sub-fs timescale in the interaction of electrons to light, the macroscopic response, namely the time delay between the arrival of a short light pulse and the appearance of the electrical output voltage on a fast linear detector is already of the order of 211 ps and for a high bandwidth photon counting device this builds up to about $1.745 \mathrm{~ns}$ (Prochazka et al. 2011), leading to a large gap with significant uncertainty between the moment of arrival of an optical signal at the detector and the actual electrical timing output response. The main dilemma is that the actual range measurements are done in the optical domain, while the detection and timing is done in the electrical domain. It is usually assumed that the conversion delay remains constant between the optical range measurement and the system calibration and therefore cancels out as a common mode effect. While this is a reasonable expectation for a controlled laboratory environment, this does not fully apply for satellite ranging, because the intensity of the return signal varies a lot from one satellite target to another and even during a single measurement sequence, as the transmission of the atmosphere can change quickly. Figure 4 illustrates this intensity dependent detector delay from an laboratory measurement. While the diagram in Fig. 4 also covers extreme intensity differences, a variation of the signal intensity of around 1 order of magnitude in the regime of low intensities (the right side of Fig. 4) are typical in satellite laser ranging. So uncompensated variable systematic errors of 10 ps and more may easily arise from intensity variations of the satellite return signal. Finally it also matters, where on the active area of the photodiode the avalanche process is seeded (Blazej and Prochazka 2009). The signal growth is faster in the center of the active area, where no restrictions are present. At the perimeter of the photosensitive area the signal build up time is longer by about 5-10 ps. Together with additional range errors caused by temporal return pulse spreading due to the geometric depth of the reflector arrays on the satellite targets, these effects currently limit the achievable accuracy and stability of the SLR technique, because these error sources are not present in the system calibration process. We note that the obtained orbit accuracy of the LAGEOS satellites currently remains at a level of about 45 ps (Appleby et al. 2016), although the precision of repeated satellite range measurements may be as low as $10 \mathrm{ps}$. A clear indication for the non-negligible effect of the discussed systematic errors is the fact that the satellite range measurements are not normally distributed, while the histograms of the calibration measurements exhibit a clean Gaussian shape. 


\subsection{Deficiencies in the Estimate of the Geometrical Reference}

Another critical process is the determination of the geometrical reference point of an SLR system. While the ranging process as such is very elegant; we have a well defined signal source and a geometrically clearly defined endpoint (the retro reflector) and the measurement process itself follows Fermat's law of the shortest path (time), it is nevertheless very challenging to cope with technically necessary compromises. These arise most prominently from limitations of the dynamic range of the measurement signals. In extreme situations one finds a difference of the order of $-170 \mathrm{~dB}$ between the generated and the detected laser pulse. As a consequence, transmit and receive functions have to be separated at the telescope. One way to overcome this difficulty is to split the signal path and use a transmit telescope different from the receive telescope or to introduce a switching unit of some making that connects the laser and the detector to the telescope in an alternating fashion. Either way, this requirement introduces a small asymmetry into the measurement process and it is not the only one. In order to establish the moment where the laser pulse passes the reference point of the SLR system on its way out to the satellite target, one has to measure the time that the laser pulse takes to travel from the laser to the telescope reference point, including the effect of cable delays and transfer times through electronic circuits on the timing of the pulse. To achieve this, SLR systems usually utilize a calibration process, which consists of repeated range measurements to a nearby geometrically flat ground target in a fixed and known position, assuming that the local signal delays are the same for both the satellite and the calibration measurement. This is almost true, but not perfectly. Since the laser signal is almost instantly back at the detector ( $\Delta \tau \approx 100 \mathrm{~ns})$, there is a small asymmetry in the ranging system electronics to allow for this. Typical satellite range measurements have a time interval of several milliseconds between laser fire and echo arrival. On top of these small differences in the signal delays between calibration and range measurement one has also to deal with similar small problems caused by the geometrical depth of the satellite target, when there is more than a single cube corner reflector involved. Variations of the signal strength of the detected laser returns are yet another complication.

\subsection{Timing Errors Affecting the Scale}

The clock frequency, when it is not representing the SI second affects the range measurement in the form of an error in scale. When the generated timescale significantly deviates from the length of the SI second and the stability of its representation is not given, it can be a limitation for the accuracy of the measurements. However, a typical commercial GPS disciplined oscillator reduces this error already to less than $1 \mathrm{~mm}$ for distances corresponding to the LAGEOS orbit. So from a clock point of view scale errors are not of concern in SLR today. Table 1 summarizes all the discussed systematic error contributions in a compact form. It is important to note that the numbers given are only rough indications, because the SLR stations differ significantly from each other with respect to design and parameter setting. For example the pulse-width of one system may be $10 \mathrm{ps}$, while for another it is more like 200 ps. Some systems use semiconductor photo-detectors with fixed threshold detection, while others use photomultipliers with constant-fraction discrimination. All these small error sources, listed in Table 1, will result in a combined measurement bias, which is neither constant over time, nor will it be the same for each station, as many parameters such as laser pulse length, electrical ground potential, instantaneous laser power and detection bandwidth vary from one observing station to the next and from one day to the other. Together with small modelling errors, reaching from small orbit model deficiencies to an 
Table 1 This table summarizes where potential systematic error sources exist in SLR systems. Since the design of the various SLR stations in the ILRS differ significantly from each other, the numerical values will also differ from one system to another

\begin{tabular}{|c|c|c|}
\hline Error source & Range & Cause \\
\hline Target depth & $\begin{array}{l}\approx 3 \mathrm{~mm}(\text { Lageos }) \\
25 \mathrm{~mm} \text { (Apollo 15) }\end{array}$ & Difference between leading edge and centroid detection \\
\hline Atmosphere & $\approx 3 \mathrm{~mm}$ & $\begin{array}{l}\text { Error in the determination of the air pressure on the ground } \\
\text { (elevation dependence) }\end{array}$ \\
\hline Photo-detection & $0-12 \mathrm{~mm}$ & $\begin{array}{l}\text { - Intensity variations (highly variable) } \\
\text { - Non-reciprocal setup between calibration and ranging (slowly } \\
\text { changing) }\end{array}$ \\
\hline Pulse shape & $\approx 2 \mathrm{~mm}$ & Non-Gaussian signal distribution \\
\hline Trigger Jitter & $\pm 2 \mathrm{~mm}$ & $\begin{array}{l}\text { - Trigger threshold jitter (lead. edge detection) } \\
\text { - Differential part of ground potential fluctuation between ranging } \\
\text { and calibration (variable with time) } \\
\text { - Near field versus far field laser pattern }\end{array}$ \\
\hline Reference Point & 0-30 mm (?) & Error in the determination of the system reference point (constant) \\
\hline Clock & - & No concern \\
\hline
\end{tabular}

unfavorable distribution of data points along the orbit for the non-linear least squares adjustment process, these measurement delays add up such, that for example today's LAGEOS orbit is established with an uncertainty of the order of $2 \mathrm{~cm}$ by the global network, although the respective precision of the averaged measurements of many SLR stations are below $5 \mathrm{~mm}$. Provided that the coherence of time can be increased such that it encompasses an entire geodetic observatory, the stable phase of the clock can be used to perform closure measurements within and across the techniques of space geodesy. This will then allow to perform accurate optical time transfer and as an additional advantage it also provides a powerful tool to establish and eradicate the otherwise not detectable systematic (delay) errors within each of the measurement techniques.

\section{Local Two-Way Time and Frequency Distribution}

\subsection{The Current Limitations for the Distribution of Time and Frequency}

Coherent time transfer from one clock to another requires a perfect control over the phase of the clock oscillator along the complete path between the two clocks who are compared. In the previous section we have discussed the technical difficulties that arise when clocks are to be compared at the level of a few ps on a single time of flight measurement. In this section we describe a way to mitigate the effect of these delays by a closed loop two-way delay compensation. The Geodetic Observatory Wettzell operates several hydrogen masers and an ensemble of 5 Cs clocks and contributes to the UTC timescale. It provides both time and frequency to all the space geodetic instrumentation of the observatory, namely 3 VLBI antennas, 2 SLR systems, several GNSS receivers and operates a dedicated time laboratory. The space geodetic instrumentation of the observatory is distributed over an area of several hectare, with 50-200 m distance between them. This means that signal lines for time and frequency have to be routed to the individual measurement devices, which introduces extra 


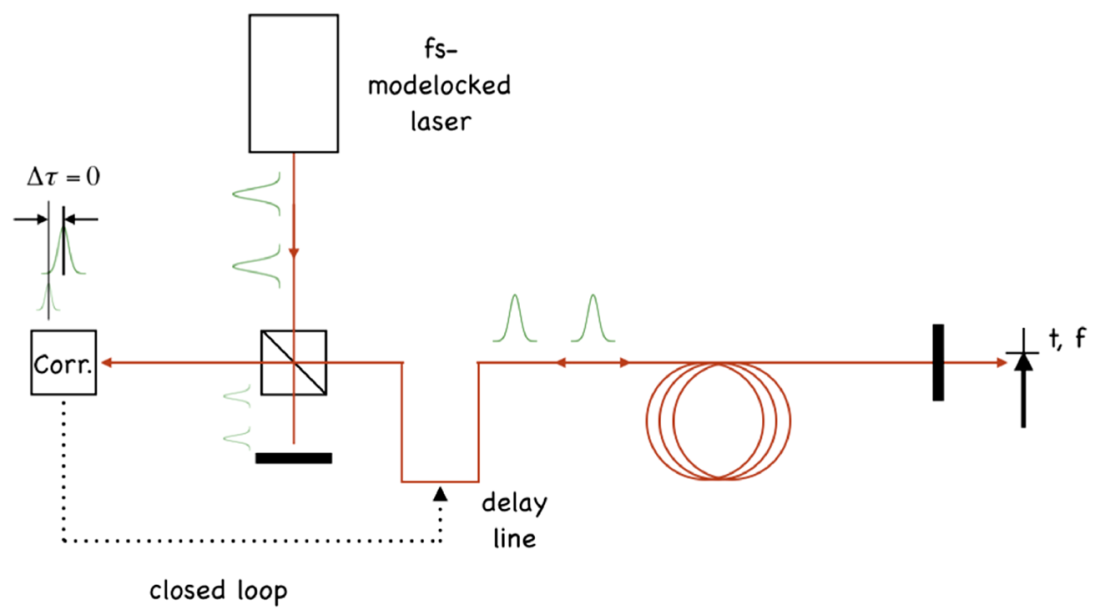

Fig. 5 Illustration of the lossless two-way time distribution system drawn in the way of an unequal arm Michelson interferometer. At the correlation stage the pulses returning from passing through the entire fiber link are superimposed on the locally reflected pulses. An actively controlled delay line is used to ensure the overlap of the local and remote pulses at all times

time delays and signal degradation artefacts, such as a reduction in signal bandwidth due to long cables for example. It also implies that we have to deal with variable extra delays in order to eventually relate the space clock to a ground clock with high accuracy. We recall that time corresponds to the integrated frequency and that the transfer of time requires a high bandwidth in order to locate the instantaneous phase with high resolution. Long cables between buildings act like a low pass filter on fast rising leading edge voltages of time markers. In the presence of different ground potentials and temperature variations in cables and even more so in the applied electronic devices through which the time signals are passing, highly variable transit delays develop, which are difficult to control. The delay between the various systems in Wettzell typically varies by as much as $1 \mathrm{~ns}$ over periods of several days with the offset in time mostly undetermined. Accurate time transfer, referenced to a distant master clock, requires a much stricter approach.

\subsection{An All Optical Two-Way Time and Frequency Distribution}

We have designed a local two-way time and frequency distribution, based on a femtosecond pulse laser, that can provide accurate time and stable reference frequencies to all measurement systems. Figure 5 illustrates the basic concept for a single link.

The reference frequency of $100 \mathrm{MHz}$ from a stable hydrogen maser is locked to the repetition rate of the mode-locked pulse laser, generating equidistant ultrashort laser pulses of less than $100 \mathrm{fs}$ pulse duration. These pulses are used to mark the zero phase instant on each cycle of the frequency. One of these pulses is associated with the beginning of the second in this time scale and this pulse is marked with an optical signal on a different wavelength as the pulse per second signal (PPS) in order to trigger all subsequent timing devices to the same timescale.

The pulse train is fed into an unequal arm Michelson type interferometer. The long arm is linking the time laboratory to the SLR facility via a fiber. A combination of the more than $100 \mathrm{~m}$ long fiber link and a coil of a conjugate fiber of the same length ensure that the pulse 
a)

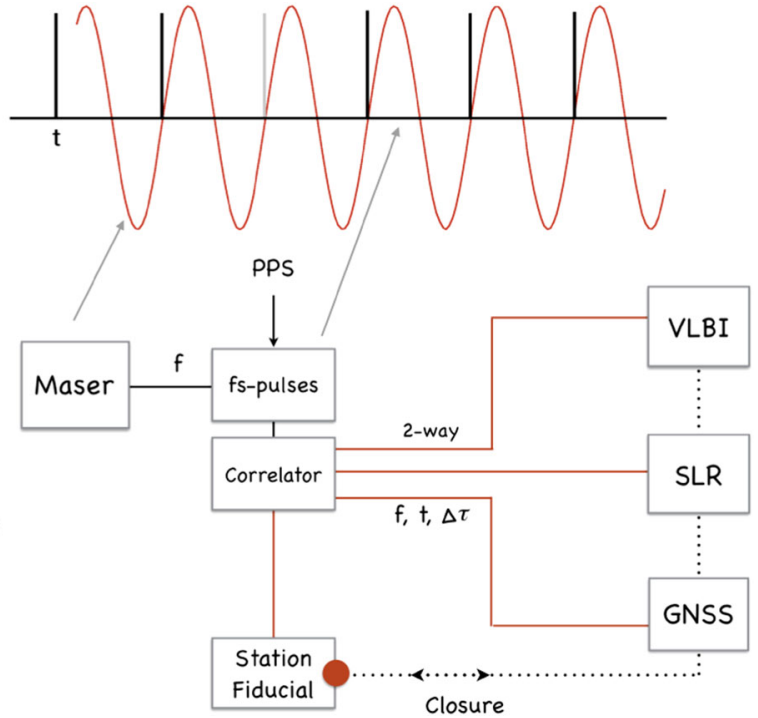

Fig. 6 The fs-mode-locked laser is locked to the stable reference frequency from the maser, so that the ultra-short laser pulses correspond to the zero-crossings of the maser frequency (a). In order to supply the measurement instrumentation the pulse train is split up in several fiber lines, each of which is stabilized by the correlation unit after a two-way passage (b). At the endpoints both, the reference frequency and the epoch can be recovered from the pulse train. The delay for each line is an integer multiple of the pulse spacing and is held constant by the feedback system of the correlator. This creates a coherent distribution of time across the observatory and provides the opportunity to apply the closure measurement technique for the reduction of systematic errors

structure is not lost due to dispersion in the fiber. At the end of the fiber a portion of the light is back-reflected towards the origin. At the input beam splitter it mixes with the pulses reflected from the short arm. The superimposed pulses are correlated and a feedback loop controls a delay line such, that the delay relationship is servoed to constant delay. This active control provides a long-term stable constant delay between both ends of the signal line. The short laser pulses generate timing signals with sub-ps resolution and from the repetition rate the clock frequency can be recovered.

Figure 6(a) sketches the full campus distribution. The pulse train from the mode-locked laser is split up in several synchronous channels, maintaining the coherence of the pulses. Each link is equipped with a balanced optical cross-correlator to compare the phase relationship of the outgoing and returning laser pulses as they are transmitted over a monomode optical fiber to the remote instrumentation (Kim et al. 2007). Each link in this star configuration is stabilized on an integer number of pulses with high accuracy. Therefore the delay introduced by each signal line is constant and known. Temperature effects on the fiber are compensated instantaneously by the feedback circuit. Since each time and frequency distribution line is independently controlled, also the delay between the various measurement systems of the observatory becomes both constant and known, thus providing the basic requirement for time transfer. The block diagram in Fig. 6(b) illustrates this setup. The VLBI, SLR and GNSS systems are all connected on individual fiber lines and obtain 4 different quantities from the time and frequency distribution unit, which are the equidistant time pips derived from the short laser pulses, the frequency of the reference oscillator contained in the repetition rate of the pulses, the beginning of a second, derived from one specifically 
Fig. 7 Time comparison between two links of the time and frequency distribution system. Each link has a fiber length of more than $100 \mathrm{~m}$ and over about one week of operation the time delay between these two terminals remains stable to within about $1 \mathrm{ps}$

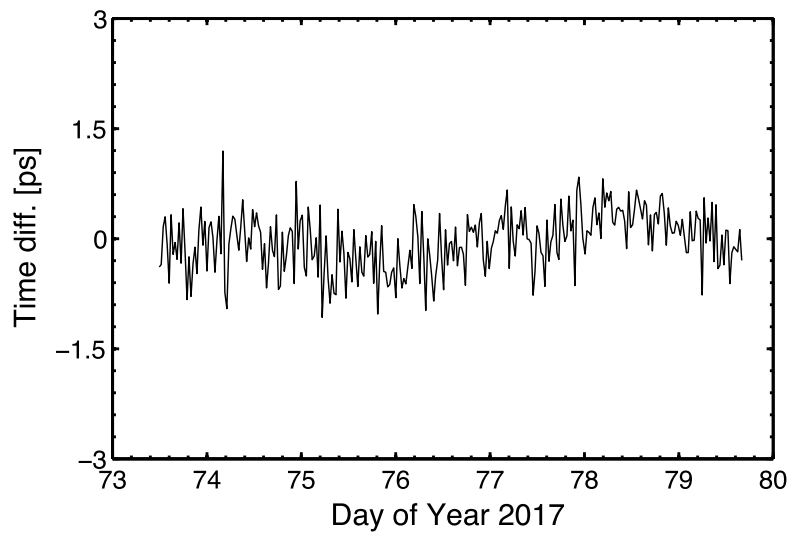

defined pulse per second out of the entire pulse train and the delay between the beginning of a new second at the central distribution unit and the accumulated delay from half the number of pulses of the roundtrip on the fiber line. Therefore it is possible to define a single timescale for the entire observatory, which is independent of any other measurement system and relates all the measurements across all space geodetic techniques to a single point of reference.

\subsection{Delay Extraction by the Virtue of Closure Measurements}

In order to define a unique reference point in time and space, we have created a local reference target for VLBI, SLR and in the future also GNSS. This geometrical reference point is also linked to the time and frequency distribution system by an independent fiber link. For SLR this means that it can be used for one-way ranging as well as for two-way ranging at the same time. In other words, it is possible to establish the accuracy of the SLR system delay by measuring the closure in time between the SLR clock and the target clock by transferring the time from the SLR system to the reference target. Any system delay that is not properly included in the SLR system calibration and that exceeds a value of a few ps, shows up as an offset between the two timing terminals and can be unambiguously identified and quantified. Furthermore it is possible to establish the long-term stability of time and frequency distribution system as well as the SLR systems on the observatory. In order to demonstrate the resolution and stability of the novel time and frequency distribution system, we have compared two independent links in different buildings more than $50 \mathrm{~m}$ apart with each other, using an asynchronous two-way time comparison system (Kodet et al. 2015). Figure 7 depicts the results. Over almost 7 days both terminals agreed to within 1 ps with each other. Owing to the active stabilization there is no temperature related drift left on these continuous time comparisons. It is important to note that the ultimate resolution for the transfer of time over these fiber links is depending on the pulsewidth of the train of pulses from the mode-locked clock laser and will be of the order of $10 \mathrm{fs}$ for the optical part of the link. However, since the time is finally accessible as an electric square wave signal at both ends of the fiber line and these electrical signals are bandwidth limited to less than $8 \mathrm{GHz}$ and in the presence of noise the practical performance limit for the resolution of the time comparison is currently at $1 \mathrm{ps}$.

Due to the controlled delay of the timing system of the observatory the laser oscillator and the VLBI source are operated phase coherently with respect to each other. This means 
that the intra-technique calibration must provide a closure with zero offset for a measurement process that starts from the common clock and leads back to the clock via the common reference target with all system delays under control. Once this is achieved, it is also possible to carry out inter-technique calibration by closure measurements from say the SLR system to the VLBI technique. Since VLBI source and SLR laser oscillator are synchronized and with constant and known delay relative to each other, erroneous system delays will become visible. Although the first several links for the delay compensated time and frequency distribution are already in place at the time of writing, the full system is far from being completed.

\section{Optical Ground to Space Time Transfer}

\subsection{Einstein Synchronization}

Giorgetta et al. have demonstrated that fs-mode-locked lasers can be used to transfer time over a fixed $2 \mathrm{~km}$ free space distance with a precision of $0.2 \mathrm{fs}$ at 1 second of integration (Giorgetta et al. 2013). Deschenes et al. have taken this concept further to a $4 \mathrm{~km}$ terrestrial distance and they could show that the long-term stability of the time transfer reached a value of $40 \mathrm{fs}$ peak to peak over two days in the presence of a turbulent atmosphere (Deschênes et al. 2016). We are setting up an all optical time transfer link between ground and the Atomic Clock Ensemble in Space (ACES) onboard the International Space Station (ISS) in space. Since the use of a fs-pulse laser is not directly applicable to the dynamic satellite orbits, we combine the fs-mode-locked laser pulses from the local timing system with the SLR technology. Due to its nature as an optical two-way time of flight measurement technique, Satellite Laser Ranging constitutes the only viable technical realization of an Einstein synchronization process (Einstein 1905). Therefore SLR provides the best suited approach for the accurate comparisons of clocks and time transfer even on intercontinental distances (Degnan 2002). In the idealized theoretical thought experiment of the Einstein publication, both the generation of the light signal as well as the detection of the presence of such a signal is instantaneous and there is no spatial offset between the light signal origin, the reflection and the clocks involved. In practice however, there are delays associated both with the generation and the detection of short laser pulses as outlined in the previous sections. There are two clocks, one of them, clock $A$ is represented in the inertial frame of a local observatory and the other $B$ is located in another inertial frame at some distance for example on an orbiting satellite. ${ }^{1}$ A short laser pulse is generated by a mode-locked laser and passes through an optical beam splitter associated with the position of the clock $A$, detecting the outgoing $\left(t_{A}\right)$ and incoming $\left(t_{A}^{\prime}\right)$ laser pulses for the synchronization process. We note that this detection process happens at an delayed instance, which is not a priori known. Figure 8, drawn in the form of a Michelson interferometer, illustrates this setup. In order to detect this transmitted light signal, a small portion of this laser pulse is passing through a reference arm of precisely known length, and after traversing the beam splitter it is detected and timed on clock $A$. The signal on the measurement branch propagates to the remote clock $B$ and is back reflected by a cube corner reflector instantaneously, so that no extra delay is introduced. A small portion of the laser beam is used to detect and time tag

\footnotetext{
${ }^{1}$ For the simplicity of arguments, we assume that the motion of the satellite clock can be approximated as a clock in another inertial frame. At the current level of measurement resolution, we may ignore the presence of rotation.
} 
Fig. 8 Illustration of the Einstein synchronization, drawn like a Michelson Interferometer. Since neither the detection process itself nor the local signal path can be realized without delay, the integration of a reference arm is needed for technical reasons. The detector delay $\tau_{1}$ cannot be compensated and has to be made as small and constant as possible

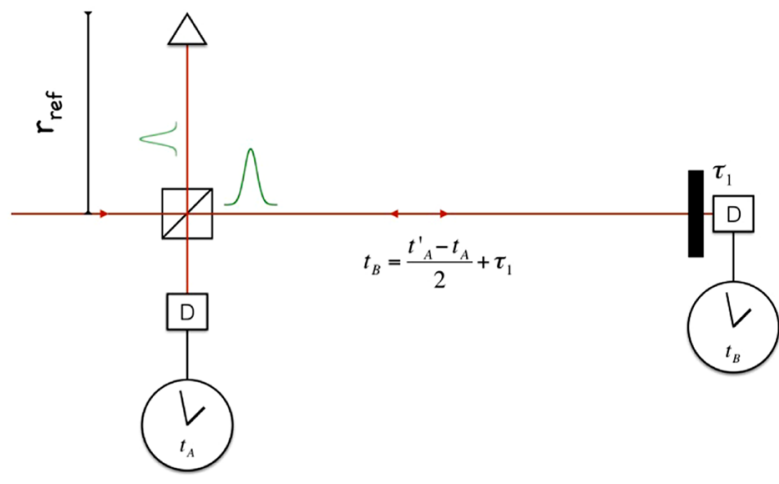

the arrival of the light signal at clock $B$. This yields the reading $t_{B}$. Again, this process is not instantaneous and the extra delay $\tau_{1}$ accounts for that. Let us design the detector such that $\tau_{1}$ is very small and that it can be established with very little uncertainty before launch. This is a reasonable assumption, ${ }^{2}$ because the remote detector requires only very few electronic components and a short signal cable to connect up to the timer. The two clocks compare as

$$
t_{B}=t_{A}+\frac{t_{A}^{\prime}-t_{A}}{2}+\tau_{1}
$$

with the delay $\tau_{1}$ stable and known. Although this concept of Einstein synchronization could in principle be applied between remote clocks on the ground, the really useful application is the clock synchronization between ground and space.

\subsection{Time Transfer Between Ground and the Atomic Clock Ensemble in Space}

One of the major objectives of the ESA project "Atomic Clock Ensemble in Space" (ACES) is the measurement of the gravitational redshift with unprecedented accuracy (Cacciapuoti and Salomon 2009). For this purpose there are two highly stable clocks provided; one is a laser cooled cold Cs-clock (primary standard) with a stability of the order of $3 \times 10^{-16}$ at one day and an accuracy of $10^{-16}$, the other is a hydrogen maser suitable for the application in space. It achieves $2 \times 10^{-15}$ in 1000 seconds. ACES will be attached to the Columbus module of the International Space Station (Hess et al. 2011). A GNSS receiver provides the orbit, so that the exact flight path of the orbiting clock can be reconstructed. The comparison between clocks on the ground and the ACES payload is achieved by Two-Way-Satellite Time and Frequency Transfer (TWSTFT) in the microwave regime. Since optical signals are neither affected by variations in the total electron content in the ionosphere nor by the variable part of water vapor in the troposphere, laser time transfer has also been adopted for this project as an independent component for the clock comparison process. Apart from more resilience of this technique against systematic errors on the propagation path, the laser time transfer method also provides a clear geometrical reference both in space and on the ground for the clock comparison process. There were two important developments required to integrate the time transfer functionality into the SLR operations. These are:

- a broadband detector onboard the satellite with an acceptance angle for the field of view of $120^{\circ}$ and a stable and well established internal signal delay;

\footnotetext{
${ }^{2}$ The discussion of Sect. 2 on variable delays due to intensity variations of the detected signal however still applies.
} 
Fig. 9 Image of the engineering module of the ACES time transfer detector. To the left one can see the wide field of view. A baffle is used to reduce the impact of the Earth albedo from the nadir direction
Fig. 10 The retro-reflector of the ELT experiment is based on the Champ/Grace design. Only a single cube corner is contributing to the back-scattered signal at any time
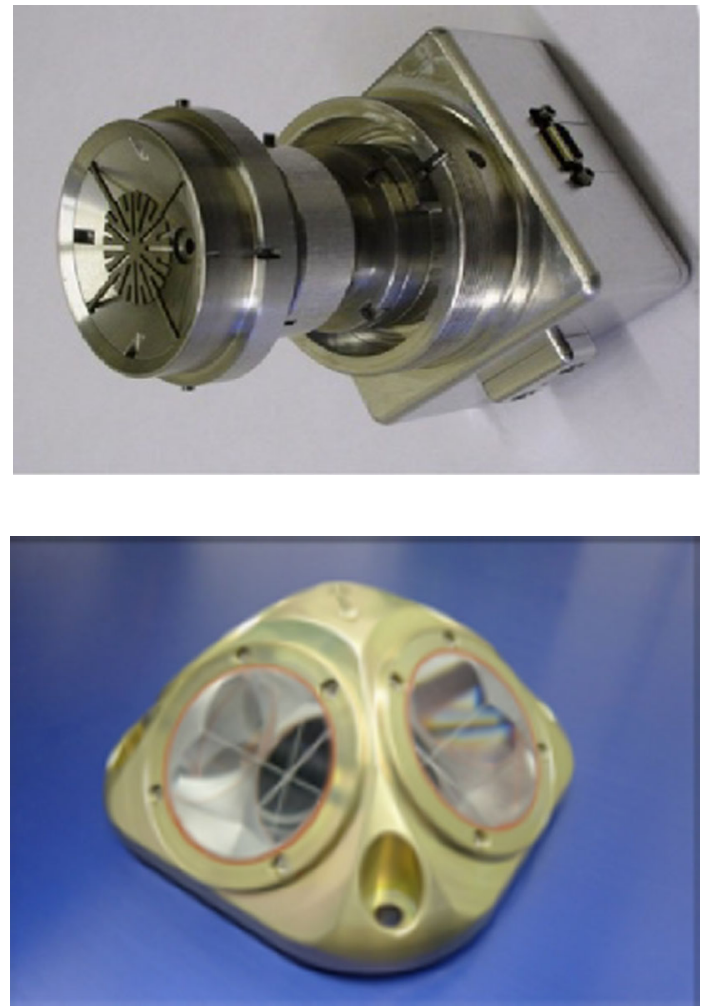

- a delay-compensated link to the local hydrogen maser, so that the SLR system is tightly referenced to the observatory clock and does not introduce extra delays, which would increase the error margin unduly.

In order to minimize the hardware requirements for the ACES payload, a solid state detector with high bandwidth and periodic self-gating was chosen. The pulse forming electronics was reduced to a minimum in order to reduce possible variations of the signal delay, which would otherwise be introduced by the detector. As a consequence, the complexity of the time transfer is shifted to the ground station, which has to fire the laser such, that the laser pulse reaches the spaceborne detector within 100-1000 ns after it is gated on. This gating process repeats with every $10 \mathrm{~ms}$ starting from the beginning of a second in the UTC timescale as it is realized aboard the ISS. In order to minimize any detector delay, the detector is capable of timing events from single photons. Therefore the scattered light from the Earth albedo is a significant noise source; in particular for a detector with such a wide acceptance angle. In Fig. 9 the engineering module of the ACES optical detector is shown. The event timing of the laser pulses at the satellite is carried out by a spare channel of the TWSTFT hardware of the ACES payload. The photo-detector is located near a small optical retroreflector array, which is shown in Fig. 10. In order to align the retro-reflector position with the detector, the eccentricity between the detector and the line of sight of the respective SLR station and reflector has to be considered. Therefore it is necessary to keep track of the attitude of the Columbus module relative to the respective SLR observatory and apply the geometrical offsets as a correction. Attitude information of the ISS is available from the downlink metrology data of the space station. Expectations are, that the optical time 
transfer will provide accurate time transfer ground to space to within $30 \mathrm{ps}$ on a single shot. At the time of writing the ACES payload is completed and awaits a suitable launch vehicle. The deployment of ACES is scheduled for 2018. In the meantime the ground segment is getting ready for the time transfer experiment. Since ELT is the first SLR operation, which involves the presence of astronauts near the target, particular safety precautions have to be met in order to guarantee that the maximum permissible energy level for pulsed laser light is not exceeded by any SLR station ranging to the ISS. The closure measurement between the SLR system and the central fiducial target of the observatory is carried out by a combination of one-way and two-way ranging. While the two-way roundtrip measurement is required to obtain the exact distance between the SLR system and the central target, the one-way range measurement is comparing the phase of the two linked clocks in order to establish a (variable) delay of the SLR system. For the establishment of the transmit system delay, the laser pulses fired from the SLR system are timed on a local detector at the central target. In a second step the fs-pulses of the timing system transmitted from the local target are detected and timed at the SLR system. This provides the receive system delay of the SLR system. Both measurements are combined to establish accurate agreement between the coherent clock signal at both locations. Consistence in these measurements and zero closure with respect to the survey of the target and the SLR station provide the desired zero offset for the time transfer between the ground clock and the ISS. The laser reflector shown in Fig. 10 is constructed in the way that only one reflector is contributing to the reflected signal. Therefore the target depth of this reflector array is comparable to a flat board. The transmit laser pulse width is as short as $10 \mathrm{ps}$ (FWHM). This leaves intensity variations and the detector delay $\tau_{1}$ (Eq. (2)) at the ISS as the main remaining contributor to the error budget of the time transfer. For the operation of the ELT time transfer it is mandatory to operate the laser at very low power, such that both for the echo detection on the ground and at the ISS the detectors work in the single photon detection regime. This minimizes the intensity depending error contribution and avoids systematic offsets between the two clocks for the comparison. The detector delay $\tau_{1}$ is calibrated prior to the launch and turns out to be very stable with a value of $0.5 \mathrm{ps} / \mathrm{K}$ with respect to temperature changes and of the order of $12 \mathrm{ps}$ (Prochazka et al. 2011, 2016). It is important to note that the variation of this predetermined value matters more than the absolute value of the delay itself. Finally the contribution of the orbit uncertainties and the attitude variation of the ISS need to be addressed. The location of the ACES clocks and the optical detector are tracked by a GNSS receiver located near the ACES clocks and the detector. From these measurements the clock motion along the orbit can be reconstructed and the necessary relativistic corrections applied. Taking all these error contributions together, it is expected that the time transfer will indeed be accurate to within $30 \mathrm{ps}$ on a single shot.

\subsection{T2L2 a Currently Existing Time Transfer Missions}

While ELT is a project still under development, another time transfer experiment has already impressively demonstrated the feasibility of the laser time transfer concept at the level of a few pico-seconds after 30 seconds of averaging. Time Transfer by Laser Link (T2L2) is in orbit as a payload on the "Ocean Surface Topography Mission", JASON 2 satellite (Samain et al. 2009). Although JASON 2 only operates an ultra-stable-oscillator (USO) as the satellite clock, all essential functions for optical time transfer can be tested by this mission (Belli et al. 2016). At the satellite the detected laser pulses are time-tagged and transmitted to the ground via a telemetry link. A signal amplitude trigger threshold ensures, that very little noise events are recorded. The first step of the evaluation process uses the laser fire and 


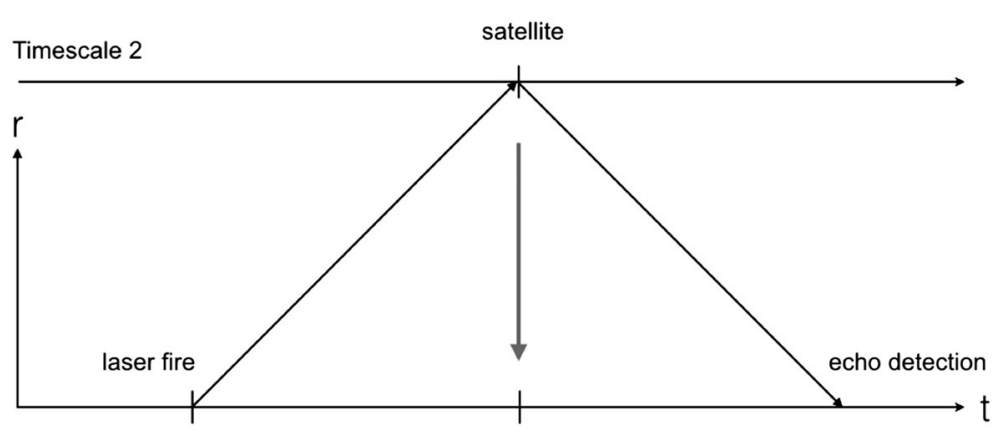

Timescale 1

Fig. 11 Illustration of the time transfer process between a ground station and a satellite. The midpoint in time between laser fire and echo detection in timescale 1 corresponds to the arrival time of the laser pulse at the satellite detector

detection events to form data triplets, containing the start and stop event on the ground station along with the detections acquired by the satellite. For this purpose the offset between the timescale on the ground and on the satellite is usually known a priori with sufficient accuracy to allow the alignment of the measurements (Samain et al. 2014, 2015). Figure 11 illustrates this relationship in a range-time-diagram. When the laser is fired, the laser pulse moves away from the ground station as time progresses. When the light hits the cube corner and the detector, it propagates back to the ground station, where it is detected, when the roundtrip is completed. For satellites in a near Earth orbit, the uplink time is equivalent to the downlink time with good approximation. Therefore the epoch corresponding to the start epoch plus $1 / 2$ of the measured roundtrip time interval in the timescale of the ground station (timescale 1) is equivalent to the detected epoch of the arrival time at the satellite in the timescale of the spacecraft, once the delay of the optical detector on the satellite is applied. Since the position of the detector and the retro-reflector on the satellite do not coincide, the respective eccentricity has to be corrected using the satellite attitude information, provided by the satellite mission. In this measurement process other systematic errors, such as for example an intensity dependent delay have either to be avoided or are required to be corrected before the respective timescales can be synchronized. For a passage of the JASON 2 satellite over the Geodetic observatory in Wettzell (Germany) the comparison of the USO oscillator on the satellite to the hydrogen maser of the observatory yielded a resolution for the time transfer of 30 ps for 1 second of averaging and 7 ps for 30 seconds of averaging (Samain et al. 2009). After 30 seconds of integration the drift of the USO became dominant. It is expected that the better ACES clocks will improve the resolution of optical time transfer further.

\section{Summary}

Small and variable system delays are currently the most obvious error sources in the techniques of space geodesy. They prohibit the use of time as an observable in space geodesy and are only visible as offsets in the measurements of the SLR, VLBI and GNSS technique. As long as time is not coherently distributed over an entire observing station, is it impossible to identify and remove the various contributors for variable system delays in the instrumentation. In the absence of any control mechanism, it must be suspected that the problem of small 
variable system delays is exacerbated by tiny asymmetries in the operation of the measurement systems and their calibration. Large and extended instrumentation like the VLBI and SLR hardware have many places where small system delays are potentially degrading the measurement quality at a subtle level. Section 2 discussed the obvious error sources in SLR. However, when the distribution of time and frequency is made coherent over the entire set of instrumentation, a novel way of delay control becomes available. Closure measurements, starting out from the coherent clock phase and passing through the entire instrumentation before coming back to the same clock in a different location, are powerful tools for systematic error identification. This however requires a stable time and frequency distribution system, where variations in the system delay are actively controlled and compensated or accurately measured. For larger observatories, where multiple techniques of space geodesy are present, the additional installation of a multi-technique ground target, connected to the station clock, offers a unique way of performing closure measurements within and across the various observation techniques. Such a target also constitutes a unique reference point for space and time, opening the door for using time as an observable in space geodesy, provided that a suitable process for clock synchronization over global distances can be established. For this purpose, optical time transfer via SLR will play an important role. In an environment, where major national standards laboratories currently develop highly accurate two-way frequency comparison techniques, in order to compare the frequency of optical clocks over distances of thousands of $\mathrm{km}$ (Lisdat et al. 2016), laser time transfer by SLR techniques can be a valuable extension on the route to extend the coherence of time over larger distances on the ground and also into the near Earth orbit. Precise clocks in space like those of the ACES project are very suitable candidates for this purpose.

Acknowledgements The authors acknowledge support from the Wettzell SLR team, namely Johann Eckl, Stefan Riepl and Günther Herold. This work was supported by the Deutsche Forschungsgemeinschaft contract SCHR 645/6-5 within the Research Group FOR1503 and by the Bundesamt für Kartographie und Geodäsie.

Open Access This article is distributed under the terms of the Creative Commons Attribution 4.0 International License (http://creativecommons.org/licenses/by/4.0/), which permits unrestricted use, distribution, and reproduction in any medium, provided you give appropriate credit to the original author(s) and the source, provide a link to the Creative Commons license, and indicate if changes were made.

\section{References}

G. Appleby, J. Rodríguez, Z. Altamimi, Assessment of the accuracy of global geodetic satellite laser ranging observations and estimated impact on ITRF scale: estimation of systematic errors in LAGEOS observations 1993-2014. J. Geod. 90(12), 1371-1388 (2016)

A. Belli, P. Exertier, E. Samain, C. Courde, F. Vernotte, C. Jayles, A. Auriol, Temperature, radiation and aging analysis of the DORIS Ultra Stable Oscillator by means of the time transfer by laser link experiment on Jason-2. Adv. Space Res. 58(12), 2589-2600 (2016). https://doi.org/10.1016/j.asr.2015.11.025

J. Blazej, I. Prochazka, Avalanche dynamics in silicon avalanche single- and few-photon sensitive photodiode. J. Phys. Conf. Ser. 193(1), 012041 (2009)

L. Cacciapuoti, C. Salomon, Space clocks and fundamental tests: the ACES experiment. Eur. Phys. J. Spec. Top. 172(1), 57-68 (2009)

J.J. Degnan, Millimeter accuracy satellite laser ranging: a review, in Contributions of Space Geodesy to Geodynamics: Technology. AGU Geodynamics Series, vol. 25 (1993), pp. 133-161

J.J. Degnan, Asynchronous laser transponders for precise interplanetary ranging and time transfer. J. Geodyn. 34(3-4), 551-594 (2002). https://doi.org/10.1016/s0264-3707(02)00044-3

J.-D. Deschênes, L.C. Sinclair, F.R. Giorgetta, W.C. Swann, E. Baumann, H. Bergeron, M. Cermak, I. Coddington, N.R. Newbury, Synchronization of distant optical clocks at the femtosecond level. Phys. Rev. X 6(2), 5109-5115 (2016)

A. Einstein, Zur Elektrodynamik bewegter Körper. Ann. Phys. 217, 891-921 (1905) 
F.R. Giorgetta, W.C. Swann, L.C. Sinclair, E. Baumann, I. Coddington, N.R. Newbury, Optical two-way time and frequency transfer over free space. Nat. Photonics 7(6), 434-438 (2013)

M.T. Hassan, T.T. Luu, A. Moulet, O. Raskazovskaya, P. Zhokhov, M. Garg, N. Karpowicz, A.M. Zheltikov, V. Pervak, F. Krausz, E. Goulielmakis, Optical attosecond pulses and tracking the nonlinear response of bound electrons. Nature 530(7), 66-70 (2016)

M.P. Hess, L. Stringhetti, B. Hummelsberger, K. Hausner, R. Stalford, R. Nasca, L. Cacciapuoti, R. Much, S. Feltham, T. Vudali, B. Léger, F. Picard, D. Massonnet, P. Rochat, D. Goujon, W. Schäfer, P. Laurent, P. Lemonde, A. Clairon, P. Wolf, C. Salomon, I. Prochazka, K.U. Schreiber, O. Montenbruck, The ACES mission: system development and test status. Acta Astronaut. 69(11), 929-938 (2011)

J. Kim, F. Ludwig, M. Felber, F.X. Kärtner, Long-term stable microwave signal extraction from mode-locked lasers. Opt. Express 15(1), 8951-8959 (2007)

J. Kodet, P. Panek, I. Prochazka, Two-way time transfer via optical fiber providing subpicosecond precision and high temperature stability. Metrologia 53(1), 18-26 (2015)

C. Lisdat, N. Quintin, C. Shi, S.M.F. Raupach, C. Grebing, D. Nicolodi, F. Stefani, A. Al-Masoudi, S. Dörscher, S. Häfner, J.-L. Robyr, N. Chiodo, S. Bilicki, E. Bookjans, A. Koczwara, S. Koke, A. Kuhl, F. Wiotte, F. Meynadier, E. Camisard, M. Abgrall, M. Lours, T. Legero, H. Schnatz, U. Sterr, H. Denker, C. Chardonnet, Y. Le Coq, G. Santarelli, A. Amy-Klein, R. Le Targat, J. Lodewyck, O. Lopez, G. Grosche, P.E. Pottie, A clock network for geodesy and fundamental science. Nat. Commun. 7, 1-7 (2016)

I. Prochazka, J. Blazej, J. Kodet, Measurement of the optical to electrical detection delay in the detector for ground-to-space laser time transfer. Metrologia 48(3), L13-L16 (2011)

I. Prochazka, J. Kodet, J. Blazej, Note: space qualified photon counting detector for laser time transfer with picosecond precision and stability. Rev. Sci. Instrum. 87(5), 056102 (2016). https://doi.org/10.1063/ 1.4948733

E. Samain, P. Exertier, P. Guillemot, F. Pierron, D. Albanese, J. Paris, J.M. Torre, I. Petitbon, S. Leon, Time transfer by laser link T2L2 first results, in The 2009 Joint Meeting of the European Frequency and Time Forum (EFTF) and the IEEE International Frequency Control Symposium (FCS) (2009), pp. 194-198

E. Samain, P. Vrancken, P. Guillemot, P. Fridelance, P. Exertier, Time transfer by laser link (T2L2): characterization and calibration of the flight instrument. Metrologia 51(5), 503 (2014)

E. Samain, P. Exertier, C. Courde, P. Fridelance, P. Guillemot, M. Laas-Bourez, J-M. Torre, Time transfer by laser link: a complete analysis of the uncertainty budget. Metrologia 52(2), 423 (2015) 